\title{
Only mass migration of fungi runs through the biotopes of soil, phyllosphere and faeces
}

\author{
Qingzhou Zhao ${ }^{1}$, Yanfen $\mathrm{Wang}^{2}$, gebiaw ayele ${ }^{3}$, Zhihong $\mathrm{Xu}^{3}$, and Zhisheng $\mathrm{Yu}^{1}$ \\ ${ }^{1}$ University of the Chinese Academy of Sciences \\ ${ }^{2}$ University of Chinese Academy of Sciences \\ ${ }^{3}$ Griffith University
}

September 28, 2020

\begin{abstract}
Understanding the microbial linkages among the soils, plants and animals is crucial for maintaining the balance of an ecosystem in grazed grasslands. However, previous studies always focused on the biotopes of soil, phyllosphere and faeces separately and little has been known about the microbial distribution and migration among these biotopes. In this study, a systematic survey to investigate the overlap and differentiation among the various microbiotas of biotopes and how the environmental filter on microorganisms served for the ecosystem was conducted at the molecular level. Our findings revealed the biotopes' role of biofilter leads to the discrepancy of microbiota distribution among the soil, phyllosphere and faeces. The substantial overlaps between soil and phyllosphere in fungi, bacteria and archaea indicated that soil could potentially perform as the microbial reservoir for phyllosphere. However, there was only fungal mass migration running through the ecosystem to link all the biotopes while there are little communal OTUs of bacteria and archaea. These findings promoted our understanding of the biotope contribution to microbial migration and improved the knowledge of microbial linkages in the grazed grassland ecosystem.
\end{abstract}

\section{Introduction}

Soils, plants and animals, as the primary objects of the grassland study, have been investigated in the traditional researches separately. Nevertheless, these issues are all the interconnected components in the entirety of grassland ecosystem. Thus, new insight of internal connections among biotopes of soil, phyllosphere and faeces in the ecosystem are urgently needed to investigate the grassland. To evaluate the components of the grassland ecosystem, the term "biotope" has been applied for a long history. The concept of biotope has evolved with quite different definitions along the development of ecology theory (Dennis, Dapporto, \& Dover, 2014; Olenin \& Ducrotoy, 2006). With the evolution of relative accompanying ideas, a new definition of the concept biotope has been accepted extensively with the contemporary meaning of the combination of substantial environment and assembled organism species (Kobori, 2009; Rodiek \& Thomas, 2007). Abiotic components are attributed to the "habitat", which means the physical and chemical environment with the distinctive physiographic features and geographical locations. The biotic components are attributed to the "community", which means groups of organism species in a particular environment with the interaction of each other or the interconnection between the organisms and environment (Dennis et al., 2014; Olenin \& Ducrotoy, 2006). Notably, compared to the entire ecosystem, Olenin and Ducrotoy pointed out that the summation of all the biotopes does not involve the energy and other linkages between the ecosystem components (Olenin \& Ducrotoy, 2006). In another words, apart from the biotopes, the connections, especially the microbial linkages among the biotopes also serve for the ecosystem running.

In a temperate grazed steppe, the ecosystem could be divided into three biotopes of great importance: soil, 
phyllosphere and faeces. All of the biotopes include the distinctive environmental features of abiotic conditions. These abiotic conditions could also regulate and control the distribution and migration of microbial community among the three biotopes. Thus, every biotope with unique abiotic and biotic characteristics is shaped into a sub-system of the pastureland ecosystem. Concurrently, all these sub-systems interact with each other by the web of food and predation. In such an environment, microbes play a role of the vector and transporter for the energy flow (Andrés et al., 2016; Bardgett \& van der Putten, 2014; Wardle et al., 2004). This function made microbiota as an important inhibitor for the environmental disturbance to regulate and maintain the ecosystem (de Vries et al., 2012).

As the main assembled organisms, soil, phyllosphere and fecal microbiota play an essential role to regulate and control the ecosystem of the grazed steppe. Initially, soil microbiota is deeply engaged participants in the grassland ecosystem. Soil microbiota is the engine of the biogenic elements in the soil-plant system to immobilize and regulate the migration and transportation of nutrient elements owing to its role of decomposer. Concurrently, soil microbiota plays a key role in the soil assimilation process to the contaminant because the microbial biotransformation is considerably correlated to the occurrence and fate of the soil contaminants (Adrian, Szewzyk, Wecke, \& Gorisch, 2000; Bunge et al., 2003; Giller, Witter, \& McGrath, 2009; Zhu, Yoshinaga, Zhao, \& Rosen, 2014). The last function of soil microbiota is regulation of the greenhouse gas emission by affecting the biogeochemical process of the elements (Brauer, Cadillo-Quiroz, Yashiro, Yavitt, \& Zinder, 2006; Erkel, Kube, Reinhardt, \& Liesack, 2006; Lu \& Conrad, 2005; Mahecha et al., 2010; Yvon-Durocher et al., 2012). Another critical microbial community controlling the grazed steppe ecosystem is phyllosphere microbiota. Phyllosphere microbiota is termed as the microbes who colonize in the aerial habitat on the surface of leaves (Steven E. Lindow \& Leveau, 2002; Vacher et al., 2016). The primary phyllosphere microbial modification is the alteration to the properties of plant surface (S. E. Lindow \& Brandl, 2003). The substantial heterogeneous aggregates of microbes also modify the traits of their microhabitat to develop the nutrient availability expression of phenotypes by the phylloplane (Horton et al., 2014; Monier \& Lindow, 2003; Rastogi, Coaker, \& Leveau, 2013; Whipps, Hand, Pink, \& Bending, 2008). The phyllosphere epiphytic microbiota modify the plant by controlling on plant disease and frost injury as well (Gourion, Rossignol, \& Vorholt, 2006; Knief, Ramette, Frances, Alonso-Blanco, \& Vorholt, 2010; Steven E. Lindow \& Leveau, 2002; Melotto, Underwood, Koczan, Nomura, \& He, 2006). In addition, the microbial adaptation regulates the resistance to the stress of the phyllosphere (Delmotte et al., 2009; Vacher et al., 2016; Vorholt, 2012; Zimmerman \& Vitousek, 2012). The last crucial compound to regulate the pasture is fecal microbiota. The fresh fecal microbiota originates from the intestinal tracts of large livestock. In a grazed grassland, $60-99 \%$ of the nutrients ingested by the herbivorous livestock are returned into soil as the excreta (Yanjiang Cai, Chang, \& Cheng, 2017). After entering to the soil through the faeces coverd patches, the fecal microbiota could regulate the decomposition process of the faeces and control the release of the nutrient element (Lin et al., 2009; Liu et al., 2018; Saggar, Bolan, Bhandral, Hedley, \& Luo, 2010). Furthermore, the physiochemical properties, biological activities and plant growth are also influenced by fecal microbiota (Y. Cai, Zheng, Bodelier, Conrad, \& Jia, 2016; Jost, Joergensen, \& Sundrum, 2012). In the pasture, the fecal microbial composition and the behavior of microbial community is a vital attribute to impact the strategy and the method of livestock utilization and management (Jost et al., 2012; Liu et al., 2018; Saggar et al., 2010). Unfortunately, there is surprisingly little knowledge about the migration and cycle of the microbial community among three substantial biotopes: soil, phyllosphere and faeces in a grazed grassland. Although the studies on soil, phyllosphere and fecal microbiota have been carried out separately, the lack of the demonstration for microbial transportation among these three biotopes limits the insight to the link among components and energy flow path in the ecosystem. Neither any study for how these microbial linkages served for the stability of the ecosystem has been made.

In this study, we investigated the distribution of soil, phyllosphere and fecal microbiota to figure out the microbial migration and cycle among these biotopes using molecular method such as high-throughput sequencing and real-time Polymerase Chain Reaction (qPCR) combining with genomic analysis and machine learning classification. The hypotheses are advanced as following: (1) Are there significant different distributions among soil, phyllosphere and fecal microbiota? (2) What are the interactions of the microbiota among 
three biotopes? and (3) What are the dominant microorganisms playing key role in this migration and cycle?

\section{Materials and Methods}

\section{Experiment design, study site and sample collection}

This study employed soil, phyllosphere and faeces samples from the same grazed grassland to compare and contrast the microbial distribution and migration among them. Samples were collected in Research Station of Animal Ecology $\left(44^{\circ} 18^{\prime} \mathrm{N}, 116 \mathrm{deg} 45^{\prime} \mathrm{E}, 1079 \mathrm{~m}\right.$ a.s.l) located in the Maodeng Pasture, Inner Mongolia Autonomous region, China. The sampling area belongs to the Xinlingol steppe with continental temperate semi- arid climate. The mean annual temperature is $3{ }^{\circ} \mathrm{C}$ and the mean annual precipitation is $350 \mathrm{~mm}$. The dominant vegetation species of the study area are Leymus chinensis (Trin.) Tzvel ,Stipa grandis, Cleistogenes squarrosa (Trin.) Keng, andAgropyron cristatum. The phyllosphere samples were collected by cutting the leaves approximately $10 \mathrm{~cm}$ above ground from $1 \mathrm{mx} 1 \mathrm{~m}$ quadrat of selected plots with 3 replicates. The corresponding soil samples were also collected in the same plots from the depth of $0-20 \mathrm{~cm}$ using a 3 $\mathrm{cm}$ diameter earth boring auger in August 2017. The excreta collected immediately from Ujimqin sheep which was the primary herbivores of the pasture was studied as the fecal samples. Another 3 replicates of phyllosphere and soil samples were collected with the same quadrat conduction in ungrazed grassland.

\section{Measurements of physicochemical properties}

The physicochemical characteristics of soil and faeces samples were measured as the Zhao et al reported (Q. Zhao et al., 2019). The phyllosphere properties were measured by the similar methods after ultrasonic leaf washing with $50 \mathrm{MHz}$ and buffer elution with potassium phosphate buffer (Zhang et al., 2008). The gravimetric water content was determined as the moisture with oven-drying at 105 for $24 \mathrm{~h}$; the $\mathrm{pH}$ was measured in the shaken soil water suspension with 1:2.5 soil: water ratio; total organic carbon (TOC) of samples was measured by a TOC5000A Analyzer (Shimadzu Corp., Kyoto, Japan) (Q. Zhao et al., 2019); total phosphate (TP) was determined with the method as previous described (Brookes, Powlson, \& Jenkinson, 1982); total carbon (TC) and nitrogen (TN) were analyzed with a Vario EL III Elemental Analyzer (Elementar, Hanau, Germany); C/N was calculated by the ratio value of $\mathrm{TC}$ and $\mathrm{TN} ; \mathrm{NO}_{3}{ }^{-}{ }^{-} \mathrm{N}$ and $\mathrm{NH}_{4}{ }^{+} \mathrm{N}$ of samples were determined by Continuous Flow Analyzer (AA3, SEAL analytical, Germany) (Z. B. Zhao et al., 2019).

\section{DNA extraction and sequencing}

Soil and fecal DNA were extracted from the collected samples using the MoBio Powersoil and Qiagen Powerfecal isolation kits according to the manufacture instructions respectively (MoBio Laboratories, Carlsbad, CA, USA). The phyllosphere genomic samples were extracted using Mobio Powersoil isolation kits after ultrasonic leave washing. Leaf samples were aseptically placed in the polypropylene tubes containing 0.1 $\mathrm{mol} / \mathrm{L}$ potassium phosphate buffer $(\mathrm{pH}$ 7.0) as the washing buffer. After the ultrasonic clean bath and the membrane filtration, the microorganisms were dislodged for the nucleic acid extraction (Zhang et al., 2008). Subsequently, a quality and quantification assessment of the purified DNA was conducted by the Nana-Drop ND-1000 Spectrophotometer (NanoDrop Technologies Inc., Wilmington, DE). The final DNA samples obtained from three biotopes were diluted and stored at $-80 \operatorname{degC}$ for further analysis.

For the Illumina Miseq High-throughput sequencing, PCR amplification of

$16 \mathrm{~S}$ rRNA were performed with universal bacterial primers 338F and 806R in the region V3-V4 (Mori et al., 2014), universal fungal primers ITS1F and ITS2R (Bokulich \& Mills, 2013) and universal archaeal primers 524F-10-ext and arch958R in the region V4-V5 respectively (Pires et al., 2012). The amplicon procceded to be sequenced on an Illumina Miseq PE250 platform.

\section{Statistical analysis}

Forward raw sequences for the amplicons were filtered by QIIME to remove the noise and chimeras. After this process, the remained sequences were clustered as operational taxonomic units (OTUs) with the average neighbor algorithm of 0.03 distance. For the libraries of bacteria and archea, the taxonomy of OTUs was 
performed using the Silva database (release 128) by RDP classifier (v2.2), while OTUs were classified with UNITE database (release 6.0) for fungal library.

For bacterial and archaeal libraries, the Silva database (release 128) was used for taxonomic assignment of OTUs by using RDP classifier (v2.2) (Wang, Garrity, Tiedje, \& Cole, 2007). For fungal libraries, OTUs were classified against the UNITE database (release 6.0).

All the statistic analysis were performed in R library using packages of "vegan", "Hmisc", "ggtern" and "ggplot2" except for the otherwise annotation. To determine the $\alpha$-diversity, Shannon and Simpson index were caculated for microbial diversity while ace and chao index for richness and shannoneven index for evenness. To identify the microbial $\beta$-diversity among soil, phyllosphere and faeces, ANOSIM, ADONIS and Polar Coordinate Analysis (PCoA) were performed by the "ANOSIM", "ADONIS" and "PCoA" function of the "vegan" package (Dixon, 2003). The OTUs overlaps were conducted into the Venn analysis using package of "venndiagramm". A network-like venn plot were generated to reflect the comparison of OTUs among three biotopes by the software of gephi. The taxa were also clustered into Hierarchical clustering tree at Order level by the "stats" package built-in R library using the algorithm of bray-curtis. To clarify the alteration of the microbial community among biotopes, the relative abundance of the microbial communities on the phylum level were presented via ANOVA barplot, radar plot and coxcomb plot. The Ternary Analysis, which visually exihbited the microbial variation among three biotopes, was presented by the "ggtern" package (Hamilton, 2017).

To examine the environmental impact on microbial community, the canonical correlation analysis (CCA) was visualized using the "CCA" function in the "vegan" package and embellished by "ggplot2" package with the environmental factors of "carbon", "nitrogen", "nitrate nitrogen", "ammonia nitrogen", "phosphorus", "temperature", "pH" and "moisture" (Dixon, 2003; Wickham, 2009). All these factors were also calculated for the Mantel test to confirm their respective influence on microbial communities through "vegan" package (Dixon, 2003). A structure equitation model (SEM) was constructed to examine the effect of physical and nutrient factor effect on microbiota among biotopes via path coefficient. SEM was commonly used as a multivariate technique achieved by statistical methods and computer algorthms (Gupta, Kapur, \& Kumar, 2017). The appropriate variables were selected based on the test for pre-assumed causal relationships as an initial step of SEM. Such test was based on the statical cariteria referring to the standard estimating results and path coefficients via Amos, 22.0 (SPSS Inc., Chicago, IL, USA) (Mardani et al., 2017). The Random Forest model was employed to present the top 15 genuses of the microbial community via random forest package in $\mathrm{R}$.

\section{Network construction}

The OTUs with relative abundance $<0.01 \%$ were removed to reduce the rare OTUs in dataset. Only the OTUs occurred in more than half of the samples were screened for the co-occurrence networks. The cooccurrence networks were constructed based on the Spearman correlation matrix with "Hmisc" package (Harrell, 2008). The nodes of the networks indicated the species and the edges between them implied the correlation between the OTUs. The P values were controlling symmetrically for the further multiple test via the false discovery rate (FDR) procedure (Benjamini, Krieger, \& Yekutieli, 2006). A cutoff wall was set up based on the Spearman correlation values and $\mathrm{P}$ values. All the cutoff of correlation coefficient were 0.9 via random matrix theory-based method. The cutoff of the $\mathrm{P}$ values for fungi and bacteria were determined as 0.001 (Luo, Zhong, Yang, Scheuermann, \& Zhou, 2006). To simplify the network for better visualization, only fungal and bacterial OTUs over than 30 and archaeal OTUs over than 5 were employed. The matrix containing the information of nodes and edges were visulized into image by Gephi platform using the algorithm of Fruchterman Reingold (Bastian, Heymann, \& Jacomy, 2009).

\section{Results}

A total of 535430 bacterial 16S rRNA V4 amplicon sequence were obtained from 9 samples covering 3 biotopes: soil, phyllosphere and faeces in the grazed temperate steppe in Inner Mongolia and further clustered into 2136 OTUs. Simultaneously, 840851 ITS gene sequence and $61482016 \mathrm{~S}$ gene sequence were also 
generated and clustered into 1340 fungal and 621 archaeal OTUs respectively.

The $\alpha$-diversity revealed distinct differences in richness, diversity and evenness of communities among biotopes of soil, phyllosphere and faeces (Table S1). The Shannon and Simpson index indicated that the soil fungal and bacterial diversity were induced in phyllosphere and faeces contrasted to the soil while fungi and bacteria showed diversity at the lowest level in faeces. The ace and chao index demonstrated the highest fungal richness in the soil than in the other two biotopes. The fungal and bacterial richness also diminished in the faeces.

All the microbiotas showed significant different distributions among the biotopes of soil, phyllosphere and faeces. The microbial communities were visualized via polar coordinate analysis (PCoA) (Fig. 1a, 1b and 1c). The PCoA graphs indicated that distinct clusters were shaped among the biotopes of soil, phyllosphere and faeces for fungal, bacterial and archaeal phyla (Fig. 1a, 1b and 1c). These clusters were confirmed by the sample within-between analysis which consisted of permutational multivariate analysis of variance (ADONIS) and similarity analysis (ANOSIM) (Table 1). These examinations also revealed that bacterial community varied among the three biotopes more significantly than fungi and archaea. The Hierarchical clustering tree at order level, based on bray-curtis distance, further supported these microbial phylogenetic differentiations among the three biotopes (Figure S1).

The network like venn analysis revealed that soil was the potential microbial source reservoir of phyllosphere while faeces was a much less relavant biotope to them. Figure. 2a indicated that phyllosphere shared large amount of OTUs with soil. However, faeces shared much fewer OTUs with the other two biotopes, especially rarely with the soil. The OTUs shared by all the three biotopes also contributed a fraction of low percentage (Fig. 2a). To be precise, the venn analysis of fungi, bacteria and archaea (Fig. 2b, 2c and 2d) showed that the main proportion of shared OTUs are contributed by fungi and bacteria. The 916 and 419 OTUs were shared by soil and phyllosphere for fungi and bacteria respectively while only 26 OTUs for archaea. However, with respect to the OTUs between soil and faeces, only 20 occurred in fungi, and both the OTUs numbers in bacteria and archaea are 0. The OTUs shared among all the three biotopes were also mainly in fungi. The three biotopes communal OTUs numbers are 104, 33 and 6 for fungi, bacteria and archaea successively.

The microbial composition also showed remarkable differentiation among the biotopes of soil, phyllosphere and archaea. For the fungi, samples from the soil biotope were more diverse than those of the phyllophere and faeces biotopes. Ascomycota (62.94\%) was the dominant phylum followed with Zygomycota (14.97\%) and Basidiomycota (10.01\%) in the soil. Ascomycota was also the most predominant phylum in phyllosphere while its relative abundance increased to $89.69 \%$ and it was $2.63 \%$ and $5.99 \%$ for Zygomycota and Basidiomycota respectively. Moreover, Ascomycota (97.36\%) almost completely dominated in the faeces fungal microbiome (Fig. 3a). For the bacterial community, the fecal microbiota was not as diverse as the soil and phyllosphere microbiota. The dominant bacterial phyla in the soil and phyllosphere were both Actionobacteria, Fimicutes, Proteobacteria, Bacteroidetes, Acidobacteria, Chloroflexi and Cyanobacteria. Actinobacteria (soil: 44.02\%, phyllosphere:34.69\%) contributed most in both biotopes. The relative abundance of Bacteroidetes (soil: 0.92\%, phyllosphere:10.99\%) and Acidobacteria (soil: 20.29\%, phyllosphere:1.83\%) increased or decreased most acutely from the soil to phyllosphere biotopes. Nevertheless, Firmicutes (61.71\%) and Bacteroidetes $(24.63 \%)$ dominated in the fecal microbiota (Fig. 3b). In the archaeal community, the dominant phyla in the soil and phyllosphere biotopes were Thaumarchaeota while Euryarchaeota dominated in the faeces biotope (Fig. 3c).

To further understand the association of the microbial structure among the soil, phyllosphere and faeces, the fungal, bacterial and archaeal composition of three biotopes were visualized by ternary analysis (Fig. 1d, 1e and 1f). A plenty of fungal classes were shared between the soil and phyllosphere with low abundance. Few fungal classes coexisted between faeces and the other two biotopes. Several fungal classes with a certain amount of relative abundance were communal among three biotopes. Most of these classes belonged to phylum Ascomycota and some classes with low abundance belonged to Basidiomycota. As for bacteria, classes shared between the soil and phyllosphere were also common while much fewer classes occurred between faeces and phyllosphere. Furthermore, there was no bacterial class between the soil and faeces. In the archaeal 
community, all the classes shared by the soil and phyllosphere belonged to Thaumarchaeota. A few classes belong to Euryarchaeota were shared by faeces and phyllosphere but it was at a low level of abundance in phyllosphere. The archaeal classes did not coexist between soil and faeces either (Fig. 1d, 1e and 1f). The radar analysis and coxcomb analysis of the microbial composition also supported these statements. It was found that Ascomycota and Basidiomycota were communal among three fungal microbiotas but the relative abundance of Basidiomycota was lower than Ascomycota (Fig. 3d and S2). The random forest model further classfied the fungal distribution among the three biotopes. The communal genuses with the top relative abundance such as unclassified Dothideales, Neostagonospora, unclassified Sporopmiaceae, unclassified Xylariales and Pyrenophora all belonged to the fungal lineages within Ascomycota (Fig. 3f).Actinobacteria, Proteobacteria and Acidobacteria were the shared bacterial phyla between the soil and phyllosphere while Bacteroidetes were shared between phyllosphere and faeces. As for archaea, Thaumarchaeota abounded in both soil and phyllosphere biotopes. Euryarchaeota abounded in faeces but it occurred rarely in phyllosphere (Fig. S2).

To identify the influence of faeces input to the soil and phyllosphere microbiota, the microbial community of soil and phyllosphere biotopes from grazed and ungrazed grassland were presented via network analysis. Multiple network topological metrics were employed to reveal the remarkable difference between the microbial assemblages of grazed and ungrazed grassland. Although the co-occurrence networks constructed by significant correlation indicated a larger proportion of bacterial and fungal OTUs were included in the networks of grazed grassland, there were similar edges in these assemblages with grazing (Fig. 4a and 4b). The average weighed degree, which indicated the normalized number of connections to a node, diminished under the grazing conditions. The decreasing cluster coefficient and increasing average path length also exhibited the attenuated connectivity of fungal and bacterial networks in the grazed grassland. Likewise, the margin attenuation of fungal and bacterial networks density under grazing conditions reflected that enormous potential connection between nodes of fungal network lost efficacy in the grazed grassland. Nevertheless, the modularity index indicated that the bacterial and fungal assemblages were more modular in the grazed grassland than ungrazed grassland while it was reverse in the archaeal assemblages (Table S2).

To clarify the influence factor of the microbiota among biotopes of the soil, phyllosphere and faeces, the environmental characteristics were determined (Table S3). The Mantel Test revealed that total nitrogen $(\mathrm{TN}), \mathrm{pH}$ and temperature influenced all the fungal, bacterial and archaeal microbiota highly significantly and total organic carbon (TOC) also influenced fungi very remarkably (Table S4). Although all the environmental factors such as TOC, TN, total phosphorus (TP), nitrate nitrogen $\left(\mathrm{NO}_{3}{ }^{-} \mathrm{N}\right)$, ammonia nitrogen $\left(\mathrm{NH}_{4}{ }^{+}-\right.$ $\mathrm{N})$, carbon nitrogen ratio $(\mathrm{C} / \mathrm{N}), \mathrm{pH}$, temperature and moisture could influence the fungal and bacterial communities, there were only TOC, TN, pH and temperature exerting impact on archaeal community. The canonical correspondence analysis (CCA) indicated that there was no predominant influence factor in these environmental variables (Fig. 5). Carbon, nitrogen and phosphorus impacts were strongly and positively correlated while they were negatively correlated to $\mathrm{pH}$ impact. Moisture and ammonia nitrogen were in another strongly positive correlated impact pair while they were significantly and negatively correlated to nitrate nitrogen. Notably, soil bacteria were most strongly influenced by nitrate nitrogen and the phyllosphere bacterial community were most correlated to temperature.

The structure equation model (SEM) was also constructed to assess the direct and indirect environmental impacts on the microbiota of the grazed grassland ecosystem which consisted of biotopes of soil, phyllosphere and faeces (Fig. 6). The multigroup modelling approaches were applied to assess the hypothesized relationships among significant environmental physicochemical factors and microbial communities. Temperature directly and strongly influenced fungal and bacterial compositions. Nevertheless, moisture and carbon were weakly correlated to fungal compositions. Nitrogen directly influenced fungal and bacterial compositions, meanwhile, it was impacted by carbon, temperature, moisture, fungal and bacterial compositions. The only factor directly linked to fungal, bacterial and archaeal compositions was $\mathrm{pH}$. The fungal impact on bacterial community was weak while there was strong correlation between bacterial and archaeal community.

\section{Discussion}


Previous studies focused on the biotopes of soil, phyllosphere and faeces in a grazed steppe ecosystem respectively while the connections of microbiota among these biotopes, which played a role to carry out the energy flow in the ecosystem, were neglected. This study investigated the diversity, structure and the interaction of the microbial communities in these biotopes at the molecular level. It provided direct evidence to reveal the microbial distribution, migration and ecosystem function of the microbiota among these biotopes.

Soil, phyllosphere and faeces all maintained distinct microbiota and the type of biotopes explaining the majority of this microbial variation. As for the $\alpha$-diversity, the soil fungal richness was higher contrasted to the fungal microbiota from the other biotopes (Table S1). This community diversity variation was likely due to the complicate and distinctive soil environment. Soil encompasses numerous microcosms to harbour distinct microbial communities rather than one single environment. Although these soil environments were limited in the only micrometer or millimeter scale, they provided niches of unique abiotic habitat and biotic communities to filter the microbial activities, diversities and compositions (Fierer, 2017). In addition, compared to soil bacteria, soil fungi were considered more adaptive to the vast different environments. Fungi were regarded as the mediators of slower carbon cycling because of their low growth rate and tolerance of low temperature and aridity (Rinnan \& Baath, 2009; Rousk \& Bååth, 2007; Sun, Li, Avera, Strahm, \& Badgley, 2017). Furthermore, the great priority effect influenced on fungi and made it easier to survive in the extensive environments (Peay, Belisle, \& Fukami, 2012). Meanwhile, fecal richness was of the lowest level in the fungal and bacterial community among all the biotopes (Table S1). The fecal microbial communities were considered similar in those residing to the livestock intestinal tract (Romero-Perez, Ominski, McAllister, \& Krause, 2011). Obviously, the extreme environment of intestinal tract limited the gut and fecal fungal and bacterial richness via filtering the microbiota with $\mathrm{pH}$, moisture and temperature stress and anaerobic environment. With respect to the $\beta$-diversity, all the microbiome also showed distinct microbial clusters among the three biotopes. This was due to the environmental filter driving the microbial community assembling in a dynamic ecosystem. Rothschild et al revealed that the environment dominated in shaping microbiome although it was only considered as a minor factor in the previous studies (Rothschild et al., 2018). The biotope preferences of the microbiota were associated with the environmental and ecological characteristics such as physicochemical properties and physiological capabilities (Bates et al., 2011; Jiao, Xu, Zhang, \& Lu, 2019; Stams \& Plugge, 2009).

Although all the three biotopes harboured distinct microbial communities from each other, the overlaps among these microbiotas revealed the microbial migration in the grazed steppe ecosystem (Fig. 2). The largest proportion of OTUs overlap occurred between soil and phyllosphere. These overlaps mainly contributed by bacteria and fungi suggested that the majority of phyllosphere bacteria and fungi originated from the soil. In another words, soil showed its potential as a microbial source reservoir of phyllophere (Zarraonaindia et al., 2015). This was consistent with the common view that phyllosphere microbiota was influenced by the microbiota of surrounding environmental source initially and then they were selected by the leaf taxa (Copeland, Yuan, Layeghifard, Wang, \& Guttman, 2015; Lajoie, Maglione, \& Kembel, 2020; Maignien, DeForce, Chafee, Eren, \& Simmons, 2014). However, the specific environmental source influencing phyllosphere microbiota most strongly depends on the growing condition of the leaf species. Each leaf shaped a unique environment to thrive for specific microorganisms (Vacher et al., 2016; Vorholt, 2012). For instance, Copeland found the phyllosphere microbiota of several annual crops mainly originated from soil microbiota in the field condition (Copeland et al., 2015). Nevertheless, on the A. thaliana leaves, the phyllosphere microbiota mirrored airborne microbiota initially in the sterile soil (Maignien et al., 2014). A small proportion of fecal OTUs also occurred in phyllosphere while most of them were at low level of abundance. Phyllosphere microbiota was colonized on the leaf surface exposed to rapidly fluctuating temperature, moisture and relatively alternative humidity and nutrient resource (S. E. Lindow \& Brandl, 2003). Thus, although the fecal microbiota originated from a specific environment with extreme stresses quite different from most of other biotopes, there were still some OTUs shared by these two biotopes since phyllosphere could offer a temporary microenvironment similar to the fecal microbiota. However, the drastic fluctuation of environment condition made phyllosphere an extreme and hostile habitat for the microbial community (S. 
E. Lindow \& Brandl, 2003). For instance, a large majority of bacterial colonist had to face threat of being washed or killed by water, peroxide and UV light (Beattie \& Lindow, 1995; Wilson \& Lindow, 1994b). The availability of nutrient source such as glucose, fructose, and sucrose were also limited for the phyllosphere epiphytic colonization (Wilson \& Lindow, 1994a). Hence, phyllosphere microbiota maintained at a low level of abundance because it had to tolerate such environment. Nevertheless, OTUs rarely occurred in both soil and faeces biotopes. Soil lacked of the drastic fluctuation of environmental condition like phyllosphere to fit the extreme environment condition of faeces. To put it another way, the rapid fluctuation of environment condition limited the growth and activity of phyllosphere microbiota, but it made phyllosphere a most extensively adaptive biotope for microbiota. Therefore, phyllosphere played the role of bridge to link the microbial communities among biotopes of soil, phyllosphere and faeces in such a grazed steppe ecosystem.

Referring to the community structure, the microbial migration among biotopes was predominated by specific taxa. ADONIS and ANOSIM described the increasing community similarity among three biotopes with the successive order of bacteria, fungi and archaea (Table 1). The similarity for bacteria was mainly contributed by the shared taxa between soil and phyllosphere. With high relative abundance, Actinobacteria and Proteobacteria occurred in both biotopes of soil and phyllosphere as the dominant phyla (Fig. 3). Whipps and Laforest-Lapointe also found $\alpha$ - and $\gamma$-Proteobacteria the most common classes within phyllosphere. However, the proximity of the relative abundance of these two phyla in phyllosphere varied from the low abundance of Actinobacteria within tree phyllosphere while the patterns of some Conifer species were consistent with our study (Laforest-Lapointe, Messier, \& Kembel, 2016; Whipps et al., 2008). Actinobacteria were the most dominant bacteria in the soil biotope to decompose the plant litters and debris (Ventura et al., 2007). In addition, the genus of Streptomyces, which belonged to Actinobacteria, played role in inhibiting the fungal pathogens within rhizosphere. It was supposed to perform the similar function in the biotope of phyllosphere (Kunova et al., 2016). Nevertheless, the bacteria in the faeces biotopes showed significant discrepancy from the other two biotopes. The dominant phyla Firmicutes and Bacteroidetes occurred rarely in the soil and phyllosphere. The observed dominance of these phyla was due to the extreme environment in the intestinal tract of livestocks. Firmicutes were resistant to harsh environmental conditions, especially the desiccation stress, through their Gram-positive cell walls and spore-forming ability (Schimel, Balser, \& Wallenstein, 2007; Van Horn et al., 2014). Bacteroidetes were the primary gateway to take advantage of glycans and carbonhydrate for the metabolism of the microbiota in the anaerobic and extreme environment in intestinal tracts (Faith, McNulty, Rey, \& Gordon, 2011; Fischbach \& Sonnenburg, 2011; Sonnenburg et al., 2010; Wu et al., 2011). In contrast to the diversity in bacteria domain, the archaea showed homogeneous within the three biotopes. Thaumarchaeota predominated in both soil and phyllosphere. This distribution was similar to the previous study in the two biotopes respectively (Fierer et al., 2012; Rinta-Kanto et al., 2016; Taffner, Cernava, Erlacher, \& Berg, 2019). Thaumarchaeota were known as a novel phylum serving for the aerobic ammonia oxidization and the nitrification in archaea independent to Crenarchaeota and Euryarchaeota (Brochier-Armanet, Gribaldo, \& Forterre, 2012; Jiao et al., 2019; Konneke et al., 2005). In the fecal archaea, Euryarchaeota replaced Thaumarchaeota as the dominant phylum. Methanobacteria and Methanomicrobia (belonged to Euryarchaeota) composed the primary assemblages. Both these classes were methanogenic archaea colonized by gut to reduce carbon dioxide into methane through present hydrogen in the intestinal tracts (Roccarina et al., 2010; van de Pol et al., 2017). Therefore, with the environmental filtering and functional selection, bacteria and archaea migrated between the biotopes of soil and phyllosphere, rather than faeces.

The only communal phyla migrating among all the three biotopes were Ascomycota and a small amount of Basidiomycota belonging to fungi. It indicated that these fungal phyla possessed robust environmental resistance and extensive environmental adaptability. In most of the previous study focusing on the fungal communities of soil, phyllosphere and faeces, Ascomycota all occupied the significant place of the dominant phyla order (Jia, Wang, Fan, \& Chai, 2018; Perazzolli et al., 2014; Yelle, Ralph, Lu, \& Hammel, 2008). In all the three biotopes of soil, phyllosphere and faeces, the Ascomycota behaviours were saprophytic and played a role in decomposing the various substrates such as plant and animal residues to transform them into nutrition for the direct usage of relative biota. Meanwhile, Nectriaceae, a parasitic and saprophytic family 
belonging to Ascomycota, generally occurred in the livestocks and monocotyledons. Nectriaceae were pivotal for maintaining the balance of the grazed grassland ecosystem because of their function of regulating the host genotypes and environments (Jia et al., 2018). Only a few sequences classified into Basidomycota were shared by all the three biotopes. Most of them belonged to Cryptococcus. Cryptococcusoccurred commonly in the animal to cause the systemic infection and its primary risk factor was HIV infection (Cafarchia et al., 2006; Idnurm et al., 2005). TheCryptococcusfrom animal faeces could be carried into other biotopes such as soil and phyllosphere with the niches of sufficiently utilizable carbon and nitrogen source (Nielsen, De Obaldia, \& Heitman, 2007). However, whether the overlap of Cryptococcus could be applied to other terrestrial area was not clear since this overlap occurred rarely in taxa classification and this result may depend on the timing of the sampling. Thus, Ascomycota was the definitely phylum migrating in the ecosystem to link all the biotopes. Yet more types of livestock should be brought into the survey to confirm this result since only sheep were investigated in this study.

Physical environmental factors explained more variation of the microbial abundance among the biotopes while nitrogen was the most remarkable nutrient source for the microbial distribution. SEM (Fig. 6) showed that temperature explained the most of the impacts on fungi and bacteria abundance while $\mathrm{pH}$ was the only factor influenced fungi, bacteria and archaea. Mantel test (Table S4) also demonstrated that temperature influenced microbial diversity significantly. This discrepancy might be owing to the environmental sensitivity of the dominant species such as Actinobacteria, Zygomycota and Thaumarchaeota, so they were replaced by more extensively adaptive and stress-tolerant phyla such as Firmicutes, Ascomycota and Euryarchaeota in the intestinal tracts with harsh environmental conditions (Egidi et al., 2019; Jiao et al., 2019; Roccarina et al., 2010; Schimel et al., 2007; van de Pol et al., 2017; Van Horn et al., 2014). Nitrogen was also found to be the vital nutrition factor revealed by SEM (Fig. 6). This was mainly caused by Ascomycota, the communal and dominant phylum migrating among all the biotopes since it was highly associated with the nitrogen immobilization (Egidi et al., 2019). In addition, Fusarium oxysporum, Fusarium solani, Cylindrocarpon tonkinense and Gibberella fujiuroii, belonging to Ascomycota, all performed function of denitrification (LevyBooth, Prescott, \& Grayston, 2014).

Although the soil showed its potential of microbial reservoir for phyllosphere, only fungi migrated among all the biotopes of soil, phyllophere and faeces. Thus, the biotopes in an ecosystem were linked by the energy flow through the fungal community. De Vries et alreported the fungal-based process and the carbon and nitrogen cycle governed led weak resilience rather than strong resistance of grassland ecosystem (de Vries et al., 2012). Our network analysis (Fig. 4) confirmed it by exhibiting the significant discrepancy between the microbial community of grazed and ungrazed grassland after long-term moderate grazing. It was also noteworthy that fungi showed the most marginal variation in the co-occurrence networks. This was consistent with the slower response of fungi due to their lower growth rate and extensively adaptability in contrast to bacteria (Bardgett \& van der Putten, 2014; Rinnan \& Baath, 2009; Rousk \& Bååth, 2007; Sun et al., 2017). Therefore, the fungal migration among biotopes made the ecosystem robust for the short-term but instable for the long-term.

\section{Conclusion}

In conclusion, this empirical study investigated the distribution and migration of the microbiota among the biotopes of soil, phyllosphere and faece through the systematic survey. Our findings demonstrated that the type of biotopes led the microbial variation. The fungal richness of soil was the highest while the fungal and bacterial richness of faeces was the lowest among the biotopes. All the biotopes showed distinct microbiota as for $\beta$-diversity and composition. Soil performed its potential of the microbial source of phyllosphere for fungi, bacteria and archaea. However, only Ascomycota belonging to fungi definitely migrated across all the three biotopes. This fungal migration made the microbial network of the ecosystem less resilient but more resistant. Overall, various biotopes of soil, phyllosphere and faeces harboured microbiotas of distinct discrepancy while soil was the microbial reservoir of phyllosphere, and the fungal migration linked all the biotopes. This study provides new insight to clarify the connection and interaction of the biotopes and better understand how to maintain the stability and sustainable of ecosystems with the ongoing ecological 
and environmental changes.

\section{Acknowledgements}

This work was supported The National Key Research and Development Program of China (2016YFC0501802).

\section{Compliance with ethical standards}

The authors declare that they have no conflict of interest.

\section{Data accessibility statement}

The Illumina Hiseq data obtained from the present study were deposited in the NCBI small read archive (SRA) data set under the study number The $16 \mathrm{~S}$ rRNA Illumina libraries obtained from the present study were deposited in the NCBI small read archive (SRA) data set under the study number PRJNA590606.

\section{Reference}

Adrian, L., Szewzyk, U., Wecke, J., \& Gorisch, H. (2000). Bacterial dehalorespiration with chlorinated benzenes. Nature, 408 (6812), 580-583. doi:10.1038/35046063

Andrés, P., Moore, J. C., Simpson, R. T., Selby, G., Cotrufo, F., Denef, K., . . Wall, D. H. (2016). Soil food web stability in response to grazing in a semi-arid prairie: The importance of soil textural heterogeneity. Soil Biol Biochem, 97 , 131-143. doi:10.1016/j.soilbio.2016.02.014

Bardgett, R. D., \& van der Putten, W. H. (2014). Belowground biodiversity and ecosystem functioning. Nature, 515 (7528), 505-511. doi:10.1038/nature13855

Bastian, M., Heymann, S., \& Jacomy, M. (2009). Gephi: An Open Source Software for Exploring and Manipulating Networks. Paper presented at the International Aaai Conference on Weblogs \& Social Media; Third International Aaai Conference on Weblogs \& Social Media.

Bates, S. T., Berg-Lyons, D., Caporaso, J. G., Walters, W. A., Knight, R., \& Fierer, N. (2011). Examining the global distribution of dominant archaeal populations in soil. ISME J, 5 (5), 908-917. doi:10.1038/ismej.2010.171

Beattie, G. A., \& Lindow, S. E. (1995). The Secret Life of Foliar Bacterial Pathogens on Leaves. Annu Rev Phytopathol, 33 , 145-172. doi:DOI 10.1146/annurev.py.33.090195.001045

Benjamini, Y., Krieger, A. M., \& Yekutieli, D. (2006). Adaptive linear step-up procedures that control the false discovery rate.Biometrika, 93 (3), 491-507. doi:10.1093/biomet/93.3.491

Bokulich, N. A., \& Mills, D. A. (2013). Improved selection of internal transcribed spacer-specific primers enables quantitative, ultra-high-throughput profiling of fungal communities. Appl Environ Microbiol, 79 (8), 2519-2526. doi:10.1128/AEM.03870-12

Brauer, S. L., Cadillo-Quiroz, H., Yashiro, E., Yavitt, J. B., \& Zinder, S. H. (2006). Isolation of a novel acidiphilic methanogen from an acidic peat bog. Nature, 442 (7099), 192-194. doi:10.1038/nature04810

Brochier-Armanet, C., Gribaldo, S., \& Forterre, P. (2012). Spotlight on the Thaumarchaeota. ISME J, 6 (2), 227-230. doi:10.1038/ismej.2011.145

Brookes, P. C., Powlson, D. S., \& Jenkinson, D. S. (1982). Measurement of Microbial Biomass Phosphorus in Soil. Soil Biol Biochem, 14 (4), 319-329. doi:Doi 10.1016/0038-0717(82)90001-3

Bunge, M., Adrian, L., Kraus, A., Opel, M., Lorenz, W. G., Andreesen, J. R., . . . Lechner, U. (2003). Reductive dehalogenation of chlorinated dioxins by an anaerobic bacterium. Nature, 421 (6921), 357-360. doi:10.1038/nature01237 
Cafarchia, C., Camarda, A., Romito, D., Campolo, M., Quaglia, N. C., Tullio, D., \& Otranto, D. (2006). Occurrence of yeasts in cloacae of migratory birds. Mycopathologia, 161 (4), 229-234. doi:10.1007/s11046005-0194-z

Cai, Y., Chang, S. X., \& Cheng, Y. (2017). Greenhouse gas emissions from excreta patches of grazing animals and their mitigation strategies.Earth Sci Rev, 171 , 44-57. doi:10.1016/j.earscirev.2017.05.013

Cai, Y., Zheng, Y., Bodelier, P. L., Conrad, R., \& Jia, Z. (2016). Conventional methanotrophs are responsible for atmospheric methane oxidation in paddy soils. Nat Commun, 7 , 11728. doi:10.1038/ncomms11728

Copeland, J. K., Yuan, L., Layeghifard, M., Wang, P. W., \& Guttman, D. S. (2015). Seasonal community succession of the phyllosphere microbiome. Mol Plant Microbe Interact, 28 (3), 274-285. doi:10.1094/MPMI10-14-0331-FI

de Vries, F. T., Liiri, M. E., Bjørnlund, L., Bowker, M. A., Christensen, S., Setälä, H. M., \& Bardgett, R. D. (2012). Land use alters the resistance and resilience of soil food webs to drought. Nat Clim Change, 2 (4), 276-280. doi:10.1038/nclimate1368

Delmotte, N., Knief, C., Chaffron, S., Innerebner, G., Roschitzki, B., Schlapbach, R., . . Vorholt, J. A. (2009). Community proteogenomics reveals insights into the physiology of phyllosphere bacteria.Proc Natl Acad Sci USA, 106 (38), 16428-16433. doi:10.1073/pnas.0905240106

Dennis, R. L. H., Dapporto, L., \& Dover, J. W. (2014). Ten years of the resource-based habitat paradigm: the biotope-habitat issue and implications for conserving butterfly diversity. J Insec Biodiv, 2 (8), 1-32. doi:10.12976/jib/2014.2.8

Dixon, P. (2003). VEGAN, a package of R functions for community ecology.Journal of Vegetation Science, 14 (6), 927-930. doi:Doi 10.1658/1100-9233(2003)014[0927:Vaporf]2.0.Co;2

Egidi, E., Delgado-Baquerizo, M., Plett, J. M., Wang, J., Eldridge, D. J., Bardgett, R. D., . . Singh, B. K. (2019). A few Ascomycota taxa dominate soil fungal communities worldwide. Nat Commun, 10 (1), 2369. doi:10.1038/s41467-019-10373-z

Erkel, C., Kube, M., Reinhardt, R., \& Liesack, W. (2006). Genome of Rice Cluster I archaea - the key methane producers in the rice rhizosphere. Science, 313 (5785), 370-372. doi:10.1126/science.1127062

Faith, J. J., McNulty, N. P., Rey, F. E., \& Gordon, J. I. (2011). Predicting a human gut microbiota's response to diet in gnotobiotic mice. Science, 333 (6038), 101-104. doi:10.1126/science.1206025

Fierer, N. (2017). Embracing the unknown: disentangling the complexities of the soil microbiome. Nat Rev Microbiol, 15 (10), 579-590. doi:10.1038/nrmicro.2017.87

Fierer, N., Leff, J. W., Adams, B. J., Nielsen, U. N., Bates, S. T., Lauber, C. L., . . Caporaso, J. G. (2012). Cross-biome metagenomic analyses of soil microbial communities and their functional attributes.Proc Natl Acad Sci USA, 109 (52), 21390-21395. doi:10.1073/pnas.1215210110

Fischbach, M. A., \& Sonnenburg, J. L. (2011). Eating for two: how metabolism establishes interspecies interactions in the gut. Cell Host Microbe, 10 (4), 336-347. doi:10.1016/j.chom.2011.10.002

Giller, K. E., Witter, E., \& McGrath, S. P. (2009). Heavy metals and soil microbes. Soil Biol Biochem, 41 (10), 2031-2037. doi:10.1016/j.soilbio.2009.04.026

Gourion, B., Rossignol, M., \& Vorholt, J. A. (2006). A proteomic study of Methylobacterium extorquens reveals a response regulator essential for epiphytic growth. Proc Natl Acad Sci USA, 103 (35), 13186-13191. doi:10.1073/pnas.0603530103

Gupta, V., Kapur, P. K., \& Kumar, D. (2017). Modeling and measuring attributes influencing DevOps implementation in an enterprise using structural equation modeling. Inform Software Tech, 92 , 75-91. doi:10.1016/j.infsof.2017.07.010 
Hamilton, N. (2017). ggtern: An Extension to 'ggplot2', for the Creation of Ternary Diagrams. Anesthesiology, 64 (64), $72-86$.

Harrell, F. E. (2008). Hmisc: Harrell Miscellaneous. 3 , 4-4.

Horton, M. W., Bodenhausen, N., Beilsmith, K., Meng, D., Muegge, B. D., Subramanian, S., .. . Bergelson, J. (2014). Genome-wide association study of Arabidopsis thaliana leaf microbial community. Nat Commun, 5, 5320. doi:10.1038/ncomms6320

Idnurm, A., Bahn, Y. S., Nielsen, K., Lin, X., Fraser, J. A., \& Heitman, J. (2005). Deciphering the model pathogenic fungus Cryptococcus neoformans. Nat Rev Microbiol, 3 (10), 753-764. doi:10.1038/nrmicro1245

Jia, T., Wang, R., Fan, X., \& Chai, B. (2018). A Comparative Study of Fungal Community Structure, Diversity and Richness between the Soil and the Phyllosphere of Native Grass Species in a Copper Tailings Dam in Shanxi Province, China. Appl Sci, 8 (8), 1297. doi:10.3390/app8081297

Jiao, S., Xu, Y., Zhang, J., \& Lu, Y. (2019). Environmental filtering drives distinct continental atlases of soil archaea between dryland and wetland agricultural ecosystems. Microbiome, 7 (1), 15. doi:10.1186/s40168019-0630-9

Jost, D. I., Joergensen, R. G., \& Sundrum, A. (2012). Effect of cattle faeces with different microbial biomass content on soil properties, gaseous emissions and plant growth. Biol Fert Soil, 49 (1), 61-70. doi:10.1007/s00374-012-0697-y

Knief, C., Ramette, A., Frances, L., Alonso-Blanco, C., \& Vorholt, J. A. (2010). Site and plant species are important determinants of the Methylobacterium community composition in the plant phyllosphere.ISME J, 4 (6), 719-728. doi:10.1038/ismej.2010.9

Kobori, H. (2009). Current trends in conservation education in Japan.Biol Conserv, 142 (9), 1950-1957. doi:10.1016/j.biocon.2009.04.017

Konneke, M., Bernhard, A. E., de la Torre, J. R., Walker, C. B., Waterbury, J. B., \& Stahl, D. A. (2005). Isolation of an autotrophic ammonia-oxidizing marine archaeon. Nature, 437 (7058), 543-546. doi:10.1038/nature03911

Kunova, A., Bonaldi, M., Saracchi, M., Pizzatti, C., Chen, X., \& Cortesi, P. (2016). Selection of Streptomyces against soil borne fungal pathogens by a standardized dual culture assay and evaluation of their effects on seed germination and plant growth. BMC Microbiol, 16 (1), 272. doi:10.1186/s12866-016-0886-1

Laforest-Lapointe, I., Messier, C., \& Kembel, S. W. (2016). Tree phyllosphere bacterial communities: exploring the magnitude of intra- and inter-individual variation among host species. PeerJ, 4, e2367. doi:10.7717/peerj. 2367

Lajoie, G., Maglione, R., \& Kembel, S. W. (2020). Adaptive matching between phyllosphere bacteria and their tree hosts in a neotropical forest. Microbiome, 8 (1), 70. doi:10.1186/s40168-020-00844-7

Levy-Booth, D. J., Prescott, C. E., \& Grayston, S. J. (2014). Microbial functional genes involved in nitrogen fixation, nitrification and denitrification in forest ecosystems. Soil Biol Biochem, 75 , 11-25. doi:10.1016/j.soilbio.2014.03.021

Lin, X., Wang, S., Ma, X., Xu, G., Luo, C., Li, Y., . . Xie, Z. (2009). Fluxes of CO2, CH4, and N2O in an alpine meadow affected by yak excreta on the Qinghai-Tibetan plateau during summer grazing periods.Soil Biol Biochem, 41 (4), 718-725. doi:10.1016/j.soilbio.2009.01.007

Lindow, S. E., \& Brandl, M. T. (2003). Microbiology of the phyllosphere. Appl Environ Microbiol, 69 (4), 1875-1883. doi:10.1128/aem.69.4.1875-1883.2003

Lindow, S. E., \& Leveau, J. H. J. (2002). Phyllosphere microbiology.Curr Opin Biotech, 13 (3), 238-243. doi:10.1016/s0958-1669(02)00313-0 
Liu, S., Zheng, R., Guo, X., Wang, X., Chen, L., \& Hou, Y. (2018). Effects of yak excreta on soil organic carbon mineralization and microbial communities in alpine wetlands of southwest of China. J Soil Sediment, 19 (3), 1490-1498. doi:10.1007/s11368-018-2149-2

Lu, Y. H., \& Conrad, R. (2005). In situ stable isotope probing of methanogenic archaea in the rice rhizosphere. Science, 309 (5737), 1088-1090. doi:10.1126/science.1113435

Luo, F., Zhong, J. X., Yang, Y. F., Scheuermann, R. H., \& Zhou, J. Z. (2006). Application of random matrix theory to biological networks.Physics Letters A, 357 (6), 420-423. doi:10.1016/j.physleta.2006.04.076

Mahecha, M. D., Reichstein, M., Carvalhais, N., Lasslop, G., Lange, H., Seneviratne, S. I., . . Richardson, A. D. (2010). Global Convergence in the Temperature Sensitivity of Respiration at Ecosystem Level.Science, 329 (5993), 838-840. doi:10.1126/science. 1189587

Maignien, L., DeForce, E. A., Chafee, M. E., Eren, A. M., \& Simmons, S. L. (2014). Ecological succession and stochastic variation in the assembly of Arabidopsis thaliana phyllosphere communities. MBio, 5 (1), e00682-00613. doi:10.1128/mBio.00682-13

Mardani, A., Streimikiene, D., Zavadskas, E. K., Cavallaro, F., Nilashi, M., Jusoh, A., \& Zare, H. (2017). Application of Structural Equation Modeling (SEM) to Solve Environmental Sustainability Problems: A Comprehensive Review and Meta-Analysis. Sustainability, 9 (10), 1814. doi:ARTN 1814

$10.3390 /$ su9101814

Melotto, M., Underwood, W., Koczan, J., Nomura, K., \& He, S. Y. (2006). Plant stomata function in innate immunity against bacterial invasion.Cell, 126 (5), 969-980. doi:10.1016/j.cell.2006.06.054

Monier, J. M., \& Lindow, S. E. (2003). Differential survival of solitary and aggregated bacterial cells promotes aggregate formation on leaf surfaces. Proc Natl Acad Sci USA, 100 (26), 15977-15982. doi:10.1073/pnas.2436560100

Mori, H., Maruyama, F., Kato, H., Toyoda, A., Dozono, A., Ohtsubo, Y., . . . Kurokawa, K. (2014). Design and experimental application of a novel non-degenerate universal primer set that amplifies prokaryotic $16 \mathrm{~S}$ rRNA genes with a low possibility to amplify eukaryotic rRNA genes. DNA Res, 21 (2), 217-227. doi:10.1093/dnares/dst052

Nielsen, K., De Obaldia, A. L., \& Heitman, J. (2007). Cryptococcus neoformans mates on pigeon guano: implications for the realized ecological niche and globalization. Eukaryot Cell, 6 (6), 949-959. doi:10.1128/EC.00097-07

Olenin, S., \& Ducrotoy, J. P. (2006). The concept of biotope in marine ecology and coastal management. Mar Pollut Bull, 53 (1-4), 20-29. doi:10.1016/j.marpolbul.2006.01.003

Peay, K. G., Belisle, M., \& Fukami, T. (2012). Phylogenetic relatedness predicts priority effects in nectar yeast communities. Proc Biol Sci, 279 (1729), 749-758. doi:10.1098/rspb.2011.1230

Perazzolli, M., Antonielli, L., Storari, M., Puopolo, G., Pancher, M., Giovannini, O., . . Pertot, I. (2014). Resilience of the natural phyllosphere microbiota of the grapevine to chemical and biological pesticides. Appl Environ Microbiol, 80 (12), 3585-3596. doi:10.1128/AEM.00415-14

Pires, A. C., Cleary, D. F., Almeida, A., Cunha, A., Dealtry, S., Mendonca-Hagler, L. C., . . . Gomes, N. C. (2012). Denaturing gradient gel electrophoresis and barcoded pyrosequencing reveal unprecedented archaeal diversity in mangrove sediment and rhizosphere samples. Appl Environ Microbiol, 78 (16), 55205528. doi:10.1128/AEM.00386-12

Rastogi, G., Coaker, G. L., \& Leveau, J. H. (2013). New insights into the structure and function of phyllosphere microbiota through high-throughput molecular approaches. FEMS Microbiol Lett, 348 (1), 1-10. doi:10.1111/1574-6968.12225 
Rinnan, R., \& Baath, E. (2009). Differential utilization of carbon substrates by bacteria and fungi in tundra soil. Appl Environ Microbiol, 75 (11), 3611-3620. doi:10.1128/AEM.02865-08

Rinta-Kanto, J. M., Sinkko, H., Rajala, T., Al-Soud, W. A., Sorensen, S. J., Tamminen, M. V., \& Timonen, S. (2016). Natural decay process affects the abundance and community structure of Bacteria and Archaea in Picea abies logs. FEMS Microbiol Ecol, 92 (7), fiw087. doi:10.1093/femsec/fiw087

Roccarina, D., Lauritano, E. C., Gabrielli, M., Franceschi, F., Ojetti, V., \& Gasbarrini, A. (2010). The role of methane in intestinal diseases. Am J Gastroenterol, 105 (6), 1250-1256. doi:10.1038/ajg.2009.744

Rodiek, J., \& Thomas, J. W. (2007). Wildlife habitat planning and landscape architecture. Landscape Res, 5 (3), 12-16. doi:10.1080/01426398008705949

Romero-Perez, G. A., Ominski, K. H., McAllister, T. A., \& Krause, D. O. (2011). Effect of environmental factors and influence of rumen and hindgut biogeography on bacterial communities in steers. Appl Environ Microbiol, 77 (1), 258-268. doi:10.1128/AEM.01289-09

Rothschild, D., Weissbrod, O., Barkan, E., Kurilshikov, A., Korem, T., Zeevi, D., . . . Segal, E. (2018). Environment dominates over host genetics in shaping human gut microbiota. Nature, 555 (7695), 7695. doi:10.1038/nature25973

Rousk, J., \& Bååth, E. (2007). Fungal biomass production and turnover in soil estimated using the acetatein-ergosterol technique. Soil Biol Biochem, 39 (8), 2173-2177. doi:10.1016/j.soilbio.2007.03.023

Saggar, S., Bolan, N. S., Bhandral, R., Hedley, C. B., \& Luo, J. (2010). A review of emissions of methane, ammonia, and nitrous oxide from animal excreta deposition and farm effluent application in grazed pastures. New Zeal J Agric Res, 47 (4), 513-544. doi:10.1080/00288233.2004.9513618

Schimel, J., Balser, T. C., \& Wallenstein, M. (2007). Microbial stress-response physiology and its implications for ecosystem function.Ecology, 88 (6), 1386-1394. doi:10.1890/06-0219

Sonnenburg, E. D., Zheng, H., Joglekar, P., Higginbottom, S. K., Firbank, S. J., Bolam, D. N., \& Sonnenburg, J. L. (2010). Specificity of polysaccharide use in intestinal bacteroides species determines diet-induced microbiota alterations. Cell, 141 (7), 1241-1252. doi:10.1016/j.cell.2010.05.005

Stams, A. J., \& Plugge, C. M. (2009). Electron transfer in syntrophic communities of anaerobic bacteria and archaea. Nat Rev Microbiol, 7 (8), 568-577. doi:10.1038/nrmicro2166

Sun, S., Li, S., Avera, B. N., Strahm, B. D., \& Badgley, B. D. (2017). Soil Bacterial and Fungal Communities Show Distinct Recovery Patterns during Forest Ecosystem Restoration. Appl Environ Microbiol, 83 (14), 00966-17. doi:10.1128/AEM.00966-17

Taffner, J., Cernava, T., Erlacher, A., \& Berg, G. (2019). Novel insights into plant-associated archaea and their functioning in arugula (Eruca sativa Mill.). J Adv Res, 19 , 39-48. doi:10.1016/j.jare.2019.04.008

Vacher, C., Hampe, A., Porté, A. J., Sauer, U., Compant, S., \& Morris, C. E. (2016). The Phyllosphere: Microbial Jungle at the Plant-Climate Interface. Annu Rev Ecol Evol System, 47 (1), 1-24. doi:10.1146/annurevecolsys-121415-032238

van de Pol, J. A., van Best, N., Mbakwa, C. A., Thijs, C., Savelkoul, P. H., Arts, I. C., . . . Penders, J. (2017). Gut Colonization by Methanogenic Archaea Is Associated with Organic Dairy Consumption in Children. Front Microbiol, 8 , 355. doi:10.3389/fmicb.2017.00355

Van Horn, D. J., Okie, J. G., Buelow, H. N., Gooseff, M. N., Barrett, J. E., \& Takacs-Vesbach, C. D. (2014). Soil microbial responses to increased moisture and organic resources along a salinity gradient in a polar desert. Appl Environ Microbiol, 80 (10), 3034-3043. doi:10.1128/AEM.03414-13

Ventura, M., Canchaya, C., Tauch, A., Chandra, G., Fitzgerald, G. F., Chater, K. F., \& van Sinderen, D. (2007). Genomics of Actinobacteria: tracing the evolutionary history of an ancient phylum. Microbiol Mol 
Biol Rev, 71 (3), 495-548. doi:10.1128/MMBR.00005-07

Vorholt, J. A. (2012). Microbial life in the phyllosphere. Nat Rev Microbiol, 10 (12), 828-840. doi:10.1038/nrmicro2910

Wang, Q., Garrity, G. M., Tiedje, J. M., \& Cole, J. R. (2007). Naive Bayesian classifier for rapid assignment of rRNA sequences into the new bacterial taxonomy. Appl Environ Microbiol, 73 (16), 5261-5267. doi:10.1128/AEM.00062-07

Wardle, D. A., Bardgett, R. D., Klironomos, J. N., Setala, H., van der Putten, W. H., \& Wall, D. H. (2004). Ecological linkages between aboveground and belowground biota. Science, 304 (5677), 1629-1633. doi:10.1126/science.1094875

Whipps, J. M., Hand, P., Pink, D., \& Bending, G. D. (2008). Phyllosphere microbiology with special reference to diversity and plant genotype. J Appl Microbiol, 105 (6), 1744-1755. doi:10.1111/j.1365-2672.2008.03906.x

Wickham, H. (2009). ggplot2: Elegant Graphics for Data Analysis . New York: Springer Science \& Business Media.

Wilson, M., \& Lindow, S. E. (1994a). Coexistence among Epiphytic Bacterial-Populations Mediated through Nutritional Resource Partitioning. Appl Environ Microbiol, 60 (12), 4468-4477.

Wilson, M., \& Lindow, S. E. (1994b). Ecological Similarity and Coexistence of Epiphytic Ice-Nucleating $($ Ice(+)) Pseudomonas-Syringae Strains and a Non-Ice-Nucleating (Ice(-)) Biological-Control Agent.Appl Environ Microbiol, 60 (9), 3128-3137.

Wu, G. D., Chen, J., Hoffmann, C., Bittinger, K., Chen, Y. Y., Keilbaugh, S. A., . . . Lewis, J. D. (2011). Linking long-term dietary patterns with gut microbial enterotypes. Science, 334 (6052), 105-108. doi:10.1126/science.1208344

Yelle, D. J., Ralph, J., Lu, F., \& Hammel, K. E. (2008). Evidence for cleavage of lignin by a brown rot basidiomycete. Environ Microbiol, 10 (7), 1844-1849. doi:10.1111/j.1462-2920.2008.01605.x

Yvon-Durocher, G., Caffrey, J. M., Cescatti, A., Dossena, M., del Giorgio, P., Gasol, J. M., . . Allen, A. P. (2012). Reconciling the temperature dependence of respiration across timescales and ecosystem types. Nature, 487 (7408), 472-476. doi:10.1038/nature11205

Zarraonaindia, I., Owens, S. M., Weisenhorn, P., West, K., Hampton-Marcell, J., Lax, S., . . . Gilbert, J. A. (2015). The soil microbiome influences grapevine-associated microbiota. MBio, 6 (2), 02527-14. doi:10.1128/mBio.02527-14

Zhang, B., Bai, Z., Hoefel, D., Tang, L., Yang, Z., Zhuang, G., . . Zhang, H. (2008). Assessing the impact of the biological control agent Bacillus thuringiensis on the indigenous microbial community within the pepper plant phyllosphere. FEMS Microbiol Lett, 284 (1), 102-108. doi:10.1111/j.1574-6968.2008.01178.x

Zhao, Q., Niu, H., Wang, Y., Cui, X., Li, Y., \& Yu, Z. (2019). Response of soil bacterial communities to moisture and grazing in the Tibetan alpine steppes on a small spatial scale. Geomicrobiol J, 36 (6), 559-569. doi:10.1080/01490451.2019.1583697

Zhao, Z. B., He, J. Z., Geisen, S., Han, L. L., Wang, J. T., Shen, J. P., . . . Zhang, L. M. (2019). Protist communities are more sensitive to nitrogen fertilization than other microorganisms in diverse agricultural soils. Microbiome, 7 (1), 33. doi:10.1186/s40168-019-0647-0

Zhu, Y. G., Yoshinaga, M., Zhao, F. J., \& Rosen, B. P. (2014). Earth Abides Arsenic Biotransformations. Annu Rev Earth Planet Sci, 42 , 443-467. doi:10.1146/annurev-earth-060313-054942

Zimmerman, N. B., \& Vitousek, P. M. (2012). Fungal endophyte communities reflect environmental structuring across a Hawaiian landscape. Proc Natl Acad Sci USA, 109 (32), 13022-13027. doi:10.1073/pnas.1209872109 


\section{Hosted file}

Figure.pdf available at https://authorea.com/users/362218/articles/483444-only-massmigration-of-fungi-runs-through-the-biotopes-of-soil-phyllosphere-and-faeces

\section{Hosted file}

Table 1.pdf available at https://authorea.com/users/362218/articles/483444-only-massmigration-of-fungi-runs-through-the-biotopes-of-soil-phyllosphere-and-faeces 